\title{
Surface Aggregation of Urinary Proteins and Aspartic Acid-Rich Peptides on the Faces of Calcium Oxalate Monohydrate Investigated by In Situ Force Microscopy
}

\author{
Matthew L. Weaver · S. Roger Qiu • \\ John R. Hoyer · William H. Casey • \\ George H. Nancollas · James J. De Yoreo
}

Received: 7 June 2008/ Accepted: 11 January 2009/Published online: 18 March 2009

(c) The Author(s) 2009. This article is published with open access at Springerlink.com

\begin{abstract}
The growth of calcium oxalate monohydrate in the presence of Tamm-Horsfall protein (THP), osteopontin, and the 27-residue synthetic peptides (DDDS) ${ }_{6} \mathrm{DDD}$ and $(\mathrm{DDDG})_{6} \mathrm{DDD} \quad(D=$ aspartic acid, $S=$ serine, and $G=$ glycine) was investigated via in situ atomic force microscopy. The results show that these four growth modulators create extensive deposits on the crystal faces. Depending on the modulator and crystal face, these deposits can occur as discrete aggregates, filamentary structures, or uniform coatings. These proteinaceous films can lead to either the inhibition of or an increase in the step speeds (with respect to the impurity-free system), depending on a range of
\end{abstract}

\footnotetext{
M. L. Weaver · W. H. Casey

Department of Chemistry, University of California, Davis, CA 95616, USA
}

M. L. Weaver

e-mail: mlweaver1@gmail.com

M. L. Weaver · W. H. Casey

Department of Geology, University of California, Davis, CA 95616, USA

M. L. Weaver · S. R. Qiu

Physical and Life Sciences Directorate, Lawrence Livermore National Laboratory, Livermore, CA 94551, USA

\section{J. R. Hoyer}

Department of Biological Sciences, University of Delaware, Newark, DE 19716, USA

G. H. Nancollas

Department of Chemistry, University at Buffalo, State

University of New York, Buffalo, NY 14260, USA

\section{J. J. De Yoreo $(\square)$}

The Molecular Foundry, Lawrence Berkeley National

Laboratory, Berkeley, CA 94720, USA

e-mail: jjdeyoreo@lbl.gov factors that include peptide or protein concentration, supersaturation, and ionic strength. While THP and the linear peptides act, respectively, to exclusively increase and inhibit growth on the (101) face, both exhibit dual functionality on the (010) face, inhibiting growth at low supersaturation or high modulator concentration and accelerating growth at high supersaturation or low modulator concentration. Based on analyses of growth morphologies and dependencies of step speeds on supersaturation and protein or peptide concentration, we propose a picture of growth modulation that accounts for the observations in terms of the strength of binding to the surfaces and steps and the interplay of electrostatic and solvent-induced forces at the crystal surface.

Keywords Biomineralization - Crystal growth .

Kinetics - Calcium oxalate monohydrate .

Tamm-Horsfall protein - Scanning probe microscopy

Kidney stones · Osteopontin

Although naturally occurring macromolecules in urine [1], such as osteopontin (OPN) and Tamm-Horsfall glycoprotein (THP), are believed to play an important role in controlling stone formation, a mechanistic understanding is missing. Numerous in vitro studies have shown that OPN strongly inhibits the nucleation and growth of calcium oxalate monohydrate - the most abundant mineral phase in urinary stones [2]. Stones are often found attached to epithelial cells and are typically aggregates of smaller crystals containing matrices of proteins, carbohydrates, and lipids [3]. A role for these macromolecular constituents in either promoting or inhibiting aggregation and/or attachment to cell membranes has been proposed [4, 5]. Indeed, some in vitro studies have concluded that THP, which is the most common protein found in human urine [6], does not inhibit 
COM crystal growth [7, 8] but acts to inhibit aggregation [9, 10] or to limit the attachment of existing crystals to cell surfaces [11-13]. Others concluded that THP plays no role in COM growth [8]. A number of studies provide direct evidence that both OPN and THP themselves readily attach to the COM surface [3, 14]. For example, using SDS-PAGE and Western blotting, Ryall et al. [15] observed that THP adhered to COM crystals taken from urine, and that "scrupulous care must be taken to ensure the complete removal of extraneous THP adventitiously associated with $\mathrm{CaOx}$ crystals ..." Using atomic force microscopy, Qiu et al. [14] found that OPN at nanomolar concentrations condensed into clusters $10-50 \mathrm{~nm}$ in diameter and $1 \mathrm{~nm}$ in height on the (101) face of COM. In another study, Sheng et al. [16], using chemical-force microscopy to investigate the binding of various functional groups to COM surfaces, found strong binding by carboxyl groups to COM surfaces, with the strongest adhesion force occurring on the (101) face.

Taken together, these observations suggest that, if these proteins play a role in promoting or inhibiting COM aggregation or attachment to cell membranes, those properties may be related to a propensity for the proteins themselves to attach to the surface. In order to clarify the effects of OPN and THP on growth kinetics, gain insight into the relationship between protein adhesion and growth modulation, and identify factors that lead to protein aggregation, we have used in situ atomic force microscopy (AFM) to investigate growth of COM in the presence of THP, OPN, and two peptides. The latter were synthetic 27residue aspartic-acid-rich linear peptides designed to act as surrogates for the aspartic-acid-rich segments of OPN, which contains subsequences characterized by runs of two and three aspartic acid residues, often separated by serine or glycine [7]. The results show that the formation of proteinaceous films and surface aggregates is a common phenomenon for aspartic-acid-rich peptides and proteins in the COM system, but that the tendency toward aggregate formation depends strongly on electrostatic interactions. Moreover, these biomolecules exhibit inhibitory or enhancing effects on kinetics that are often counter-intuitive based on the structure of the crystal face and the charge state of the peptide or protein. But regardless of whether the impact on kinetics is enhancing or inhibitory, these surfaceadsorbed proteins can be expected to have a significant impact on crystal aggregation and attachment to cells.

\section{Materials and Methods}

Peptide Synthesis and Protein Purification

Aspartic-acid-rich peptides with serine or glycine spacers were synthesized according to standard procedures $[17,18]$ by sequential addition of Fmoc amino acids. Following synthesis, the peptides were purified and molecular weights verified by mass spectrometry as described previously [18]. The condensed formulae for the peptides are (DDDS) ${ }_{6}$ DDD and (DDDG) ${ }_{6} \mathrm{DDD}$, where $D=$ aspartic acid, $S=$ serine, and $G=$ glycine. The molecular masses of the peptides were 2,955 and $2,775 \mathrm{Da}$ for (DDDS) ${ }_{6} \mathrm{DDD}$ and (DDDG) ${ }_{6} \mathrm{DDD}$, respectively, as determined by mass spectrometry and agreed with the expected values to within $99.998 \%$.

OPN was isolated by immunoaffinity purification as described [19]. Aliquots of OPN solutions were stored at $80^{\circ} \mathrm{C}$ before use. THP was isolated from $24-\mathrm{h}$ collections of normal human urine obtained with $0.01 \%$ thimerosal added as a preservative. Isolation procedures were monitored by quantitative analysis of THP concentrations performed by ELISA using a polyclonal rabbit anti-THP as the primary antibody [20]. Purification from whole urine followed the procedure originally described by Tamm and Horsfall [21] by raising the salt concentration to $0.58 \mathrm{M}$ by the addition of $\mathrm{NaCl}$, mixing, and storing the mixture overnight at $4{ }^{\circ} \mathrm{C}$ before centrifugation at 5,000 $\mathrm{g}$ for $30 \mathrm{~min}$. The precipitate of THP was washed twice with $0.58 \mathrm{M} \mathrm{NaCl}$ and centrifuged after each wash. After dissolving THP by the addition of deionized water and centrifuging, the pellet was discarded and the supernatant was dialyzed against deionized water containing $0.02 \%$ sodium azide and then precipitated again by raising the $\mathrm{NaCl}$ concentration to $0.58 \mathrm{M}$. These steps were repeated so that each sample of THP was precipitated at least five times before the final dialysis against deionized water containing $0.02 \%$ sodium azide.

With the exception of our own work [7], all published studies of calcium oxalate crystallization have used THP obtained through standard salt-precipitation method. The THP obtained through salt precipitation (spTHP) [21] migrates in a single band in SDS-PAGE gels. Immunoassays confirm that this standard method achieves only partial depletion of THP from urine; previous studies, therefore, did not evaluate the full urinary repertoire of THP molecules. Therefore we also prepared immunoaffinity-purified protein (apTHP). The supernatant after the initial salt precipitation of THP from urine was partially fractionated by adsorption to DEAE-cellulose, batcheluted, and dialyzed against a $0.05 \mathrm{M}$ Tris, $0.05 \mathrm{M} \mathrm{NaCl}$, $\mathrm{pH} 7.3$, buffer prior to immunopurification as previously described [7]. Monoclonal anti-THP beads ( $5 \mathrm{mg} \mathrm{IgG/ml}$ Sepharose beads) were prepared as previously described [7]. The B1 fractions of urinary proteins [7] were exposed to anti-THP beads in a Kontes Flex Column by slow circular rotation for $30 \mathrm{~min}$. The anti-THP beads were then extensively washed with Tris buffer prior to elution with $0.05 \mathrm{M}$ diethylamine, $\mathrm{pH}$ 11.5. Eluates were neutralized 
and then dialyzed against deionized water containing $0.02 \%$ sodium azide. In SDS-PAGE gels, this apTHP migrates in the same position as the spTHP and, also, in an additional band that migrates $\sim 10 \mathrm{kD}$ farther. (In addition to apTHP, proteins with other functional activities have also been shown to fully share a primary peptide sequence with THP; these proteins, or subfractions of THP, were also isolated by methods other than salt precipitation $[22,23]$.)

\section{COM Crystal and Solution Preparation}

The COM crystals used for these experiments were grown in vitro using a gel method [24]. A schematic showing their typical habit is given in Fig. 1. For in situ AFM experiments in aqueous solution, $\mathrm{CaC}_{2} \mathrm{O}_{4} \cdot \mathrm{H}_{2} \mathrm{O}$ solutions (calcium-to-oxalate ratio $=1$ ) were prepared using reagent-grade $\mathrm{K}_{2} \mathrm{C}_{2} \mathrm{O}_{4}$ and $\mathrm{CaCl}_{2} \cdot 2 \mathrm{H}_{2} \mathrm{O}$ dissolved in distilled deionized $(18 \mathrm{M} \Omega$ ) water. The calcium and oxalate concentrations were varied from 0.1 to $0.4 \mathrm{mM}$, corresponding to a range of supersaturation from -0.2 to 1.5. Supersaturation is defined as

$\sigma=\frac{1}{2} \ln \left[\frac{a_{\mathrm{Ca}^{2+}} \cdot a_{\mathrm{C}_{2} \mathrm{O}_{4}^{2-}}}{K_{\mathrm{sp}}}\right]$

where $K_{\mathrm{sp}}$ is the solubility product for $\mathrm{COM}$ at room temperature and was determined from the ionic activities at which step speed was equal to $0\left(K_{\mathrm{sp}}=1.66 \times 10^{-9} \mathrm{M}^{2}\right.$ [25]). The ionic activities, $a_{\mathrm{Ca}^{2+}}$ and $a_{\mathrm{C}_{2} \mathrm{O}_{4}^{2-}}$, are calculated by successive approximation of the ionic strength using the Davies equation and mass balance expressions for total calcium and total oxalate with appropriate equilibrium constants.

The concentrations of proteins used in this study were chosen according to physiological ranges. THP concentrations ranged from 10 to $100 \mathrm{nM}$, which is on the low end of the physiological range $[8,20]$. OPN concentrations ranged from 1 to $25 \mathrm{nM}$, which is similar to the physiological range. Peptide concentrations were chosen based on the magnitude of their effects over the supersaturation range used for the protein studies. Concentration ranges of 2 to $10 \mathrm{nM}$ and 40 to $200 \mathrm{nM}$ were used for (DDDS) ${ }_{6} \mathrm{DDD}$ and (DDDG) $)_{6} \mathrm{DDD}$, respectively.

The $\mathrm{pH}$ of all solutions was adjusted to $7.00 \pm 0.05$ before each experiment using a calibrated glass electrode and $\mathrm{KOH}$. The ionic strength of the growth solutions was adjusted to 0.05 or $0.15 \mathrm{M}$ by the addition of $\mathrm{KCl}$. Ionic strength, I, is defined as

$I=0.5 * \sum_{i}^{n} c_{i} z_{i}^{2}$

where $z_{i}$ and $c_{i}$ represent the ionic charge of each 'ith' species and the concentration of each species, respectively. Inductively coupled plasma atomic-emission spectroscopy was used to confirm the purity of all chemicals.

\section{In Situ AFM Imaging}

AFM observations were made on dislocation hillocks of the (101) and (010) faces [14] (Fig. 1). All in situ images were collected in contact mode (Nanoscope E or IIIA; Veeco Instruments, Santa Barbara, CA) on crystals anchored inside an enclosed fluid cell. $\mathrm{SiN}_{4}$ cantilevers and tips were used in all rate studies. The temperature was held constant at $25^{\circ} \mathrm{C}$ using a flow-through Peltier device. The images were obtained while supersaturated solutions flowed through the system. The flow rate $(\sim 2 \mathrm{ml} / \mathrm{min})$ was adjusted to ensure that growth was limited by surface kinetics and not by diffusion [26]. That is, at a given supersaturation, the step speed did not change when the flow rate was increased [27].
Fig. 1 Schematic of a synthetic COM crystal showing the three expressed faces and crystallographic axes, along with AFM images of typical hillocks on the two major faces

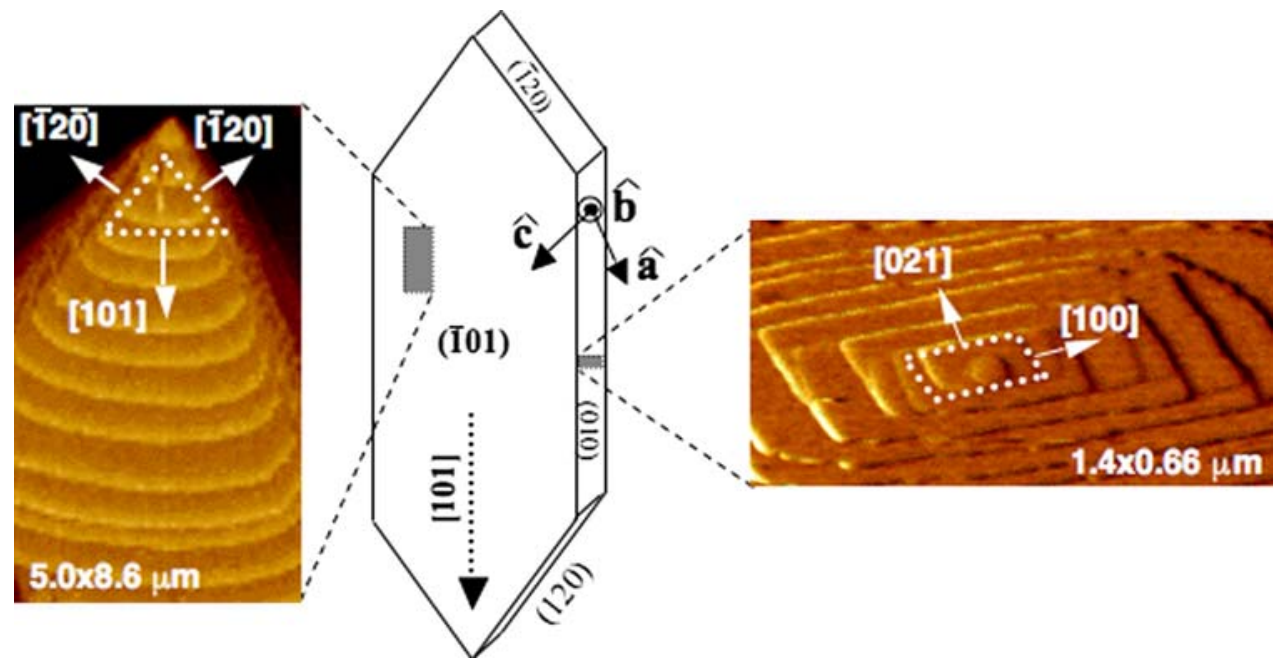


Several precautions were taken to ensure that the AFM images were authentic representations of the surface morphology and step velocities. First, the imaging force was reduced to the minimum possible value that allowed the tip to remain in contact with the surface so that there was no measurable effect of tip movement on the growth kinetics. The absence of an effect was verified by gradually increasing the force until effects on morphology and step speed were observed, by reducing the force until the tip pulled away from the surface, or by zooming out to a larger scan box and looking for the signature of the smaller scan area. A consequence of imaging in this regime is that the tip can transiently detach from the surface. The image quality is diminished slightly but the light force exerted on the sample by the tip ensures that the morphology and kinetics of the steps are minimally affected. Next, images were collected at different scan angles, and both trace and retrace images were regularly compared. This procedure eliminated the possibility that imaging artifacts arose from tip contamination or from the tip sticking to the surface. Furthermore, step speed was estimated from images that were collected at a large and a small scale to ensure that there was no effect from scanning. Because the images were taken in real time, it is inevitable that distortion of the step orientation will exist in the images. Such distortion is caused by step movement during the finite scan time, so that the apparent step orientation differs from the true orientation. The images reported here were not corrected for this effect. In fact, we use this change in angle to extract the step velocities under certain growth conditions [27].

\section{Results}

\section{Tamm-Horsfall Protein}

Figure 2 shows the temporal evolution of the surface morphology on the (101) face following introduction of an apTHP-containing solution. Although the hillock shape remained unchanged, aggregates of THP adsorbed onto the face. At a sufficiently high protein concentration, the face became completely covered. Typically, the aggregates took on an irregular filamentary shape with a dominant orientation along the (010) direction (parallel to the [101] step edge).

The time at which proteins began to be visible on the surface depended on the protein concentration. For example, at a concentration of $10 \mathrm{nM}$, the minimum time required for apTHP to be observable on the face was about $27 \mathrm{~min}$. In contrast, when a supersaturated solution containing $100 \mathrm{nM}$ apTHP was introduced, aggregates were visible on the (101) surface as soon as imaging began ( $\sim 1 \mathrm{~min})$. As shown in Fig. $2 \mathrm{~d}$, the surface became completely covered with protein after only $4-5 \mathrm{~min}$. Surprisingly, although the surface was fully covered with apTHP, the triangular growth hillock is still clearly discernible. In fact, up until the time when the steps were no longer visible, the shape of the step edges was preserved, and the step edges in all three directions were still advancing with no obvious change in speed.

In contrast to what was observed on the (101) face, aggregates did not form on the (010) face following
Fig. 2 Growth hillock on the (101) face of COM after a 0 min, b $1.5 \mathrm{~min}, \mathbf{c} 3 \mathrm{~min}$, and d $4.5 \mathrm{~min}$ of exposure to $100 \mathrm{nM}$ apTHP. Horizontal dimension of images $=3.5 \mu \mathrm{m}$. The labeled arrow in a indicates the [101] direction
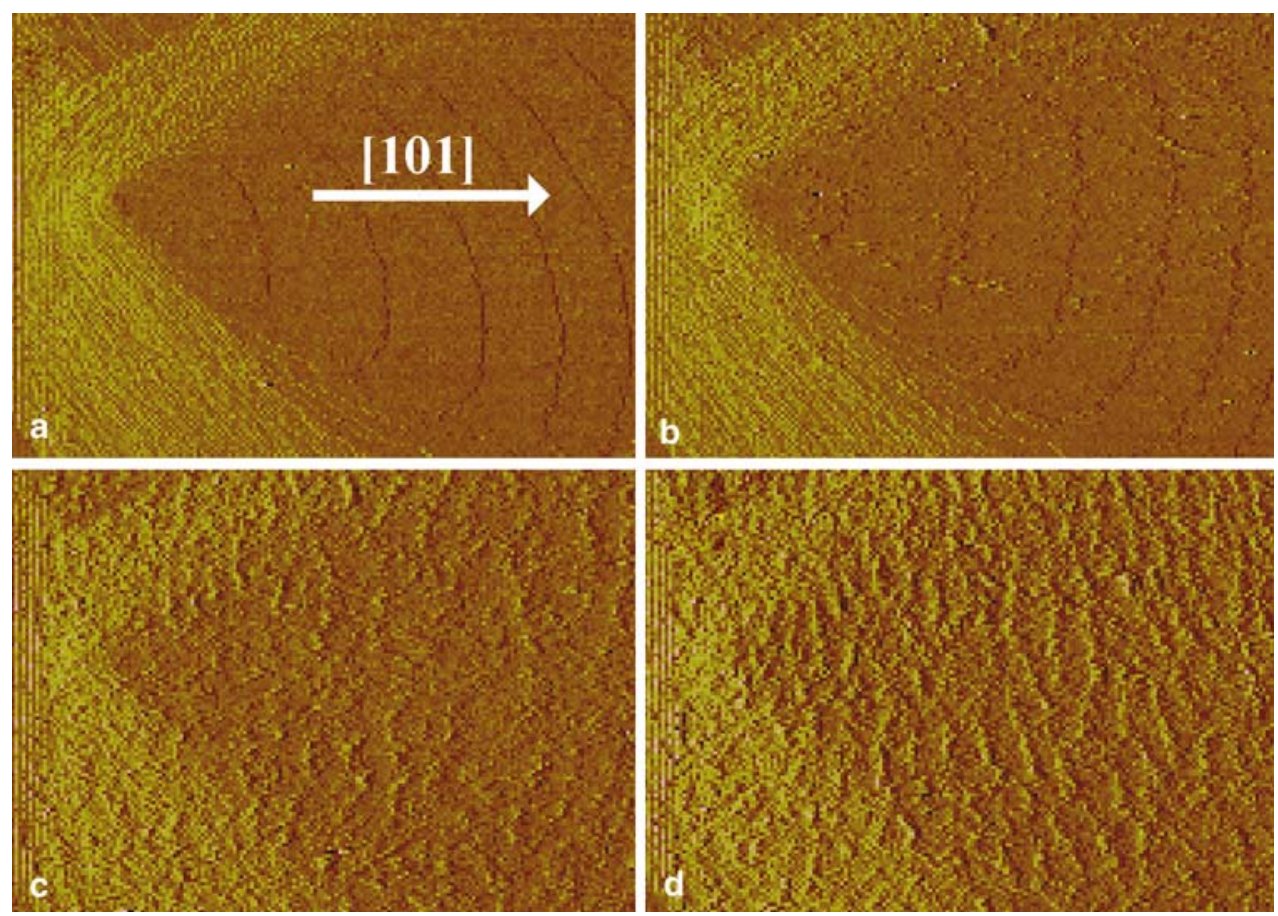
Fig. 3 Growth hillock on the (010) face of COM after $1 \mathrm{~h}$ of exposure to a $0 \mathrm{nM}, \mathbf{b} 10 \mathrm{nM}$, c $25 \mathrm{nM}$, and $\mathbf{d} 75 \mathrm{nM}$ apTHP. As apTHP concentration increases, step edges become difficult to resolve. The loss of resolution does not prevent measurement of step speed. Horizontal dimension of images $=1 \mu \mathrm{m}$. Labeled arrows in a indicate the [100] and [021] step directions
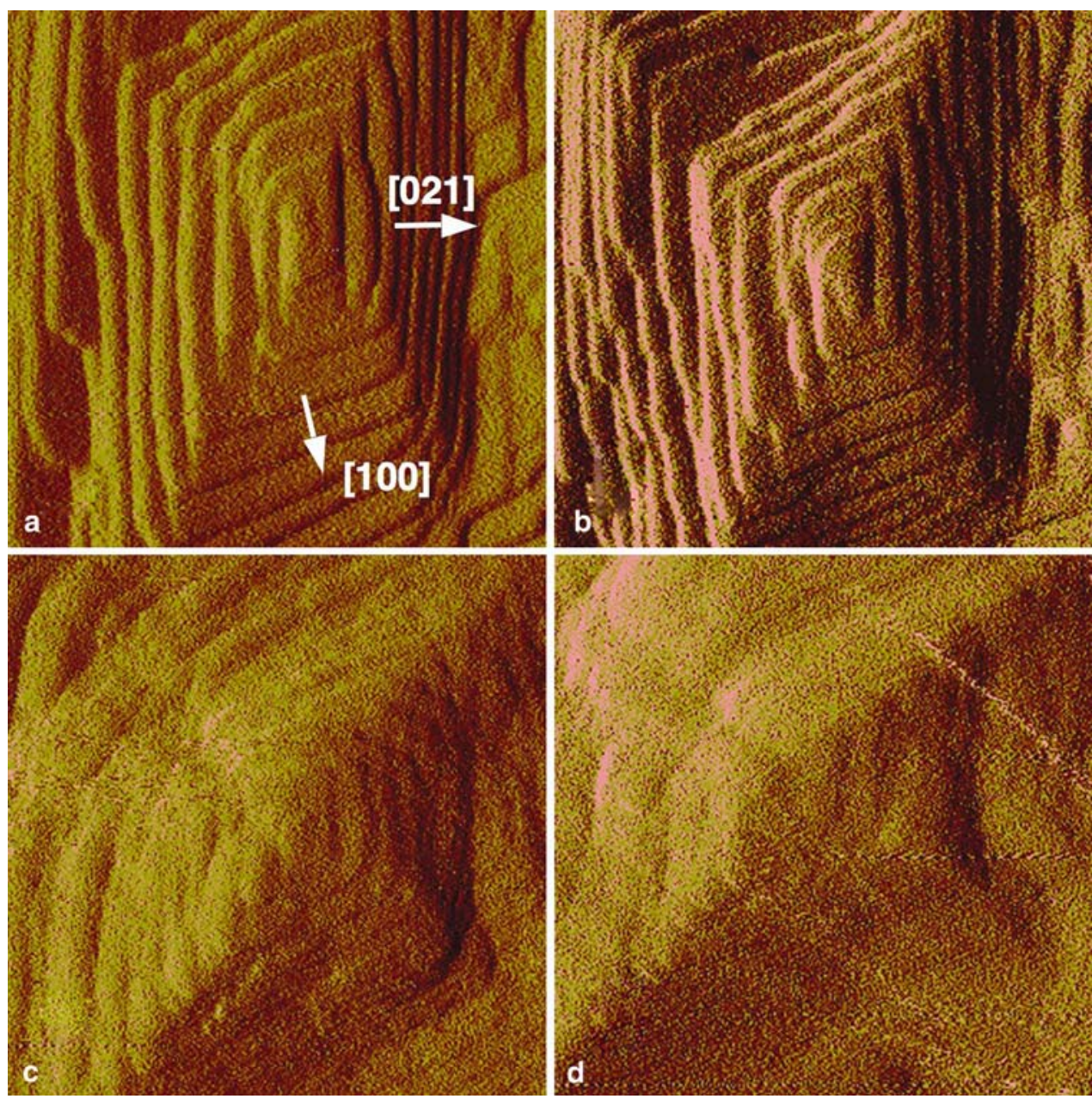

introduction of apTHP solution. The absence of aggregates on the (010) surface is shown in Fig. 3, which displays the change in growth hillock morphology with increasing protein concentrations. However, with increasing THP concentration, the steps became increasingly less distinct (Fig. 3c, d). The only reasonable conclusion we can draw from these changes is that the surface became covered in a thin film of THP. As in the case of the (101) surface, even at the highest THP concentrations, this film had only a minor impact on the step morphology or speed.

Measurements of the step speeds on the (101) face as a function of supersaturation and protein concentration show a result that is counter-intuitive. The growth kinetics were enhanced even when the face was completed coated by THP aggregates and the steps difficult to resolve. This enhancement is exhibited in Fig. 4, which shows the measured speed of the [101] step as a function of solution supersaturation at two different protein concentrations and ionic strengths. The trendlines are best fits to each set of data and show that, for both ionic strengths, while the equilibrium $\mathrm{Ca}^{2+}$ activity (i.e., the $\mathrm{Ca}^{2+}$ activity at which the step speed is zero) remained unchanged, the slope was increased by $20 \%$ to $30 \%$.

The effects of apTHP on growth kinetics on the (010) face differed from those seen on the (101) face. As Fig. 5a and $b$ show, the results obtained for the two types of steps on that face at an ionic strength of $0.05 \mathrm{M}$ were similar for both step types. They exhibited a dual functionality, though the magnitudes of the effects and the crossover points from inhibition to acceleration differed somewhat. At low calcium activities, there was either no measurable effect $(10 \mathrm{nM})$ or a slight inhibition $(25$ and $75 \mathrm{nM})$. As supersaturation was increased, the step speed increased for all three protein concentrations, leading to enhanced growth rates. However, the magnitude of the acceleration did not scale proportionally with the concentrations of protein in solution. Some, if not most, of this lack of scaling was undoubtedly due to experimental error inherent in the data, since we were measuring changes of only a few tenths of a nanometer per second. As the inset in Fig. 5b shows, at the higher ionic strength of $0.15 \mathrm{M}$, the results differ slightly in that no inhibition was observed at the apTHP concentration 


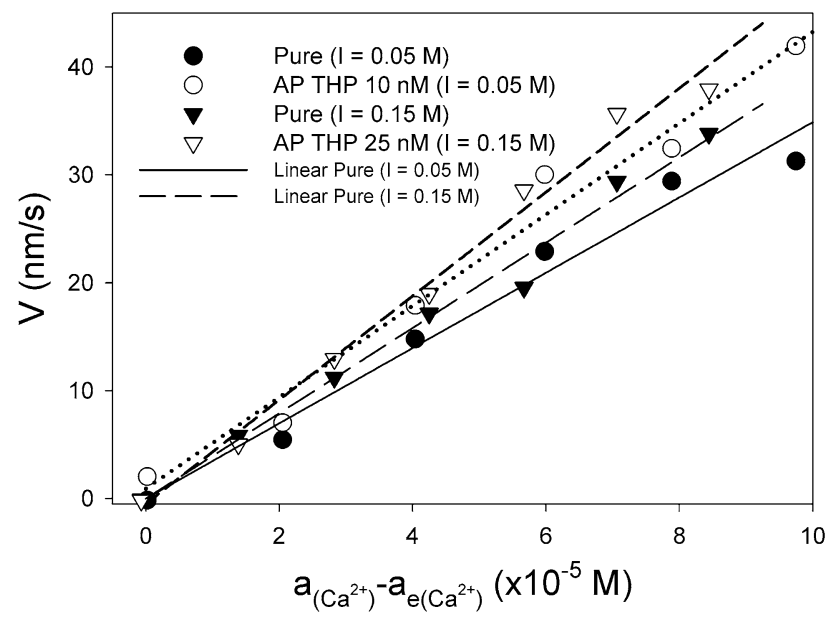

Fig. 4 The effect of apTHP on the [101] step of the (101) face of $\mathrm{COM}$ at ionic strengths of 0.05 and $0.15 \mathrm{M}$. The slope at $0.15 \mathrm{M}$ ionic strength is higher than that at $0.05 \mathrm{M}$ ionic strength. At both ionic strengths the addition of apTHP to the growth solution increases the step speed with respect to the pure system

of $25 \mathrm{nM}$ used to collect the data. However, the shapes of the curves and the magnitude of the acceleration at higher calcium activities were very similar.

The effect of spTHP on the growth kinetics of the (010) face at ionic strength of $0.05 \mathrm{M}$ was also examined for different protein concentrations. As shown in Fig. 6a, for the [021] step the dual functionality was still present, but the trend was reversed. There was slight acceleration at low calcium activity and inhibition at higher levels. However, again the magnitude of the changes in step speed did not scale proportionally with protein concentration. As with apTHP, some of this can be attributed to scatter in the data. For the [100] step, the trends are similar, though at the highest activities the data show a mix of slight inhibition and acceleration depending on the spTHP concentration (Fig. 6b). In particular, at the highest supersaturations, all protein levels gave enhancement except $75 \mathrm{nM}$, where inhibition dominated.

\section{Osteopontin}

As Fig. 7 shows, introduction of OPN leads to the formation of aggregates on the (101) face. That figure also shows how the coverage of aggregates varies with time for concentrations of 2 and $5 \mathrm{nM}$. At a concentration of $2 \mathrm{nM}$, the aggregates start out as discrete clusters $10-25 \mathrm{~nm}$ in diameter. With time, these clusters grow in length to form elongated structures with a dimension along the short axis remaining near $\sim 25 \mathrm{~nm}$ and a long axis that trends along the [010] direction, as was the case with THP. (Compare Fig. 7c, d with Fig. 2c.) At an OPN level of $5 \mathrm{nM}$, the density of the aggregates eventually becomes so high that, as with THP, the steps are no longer visible.
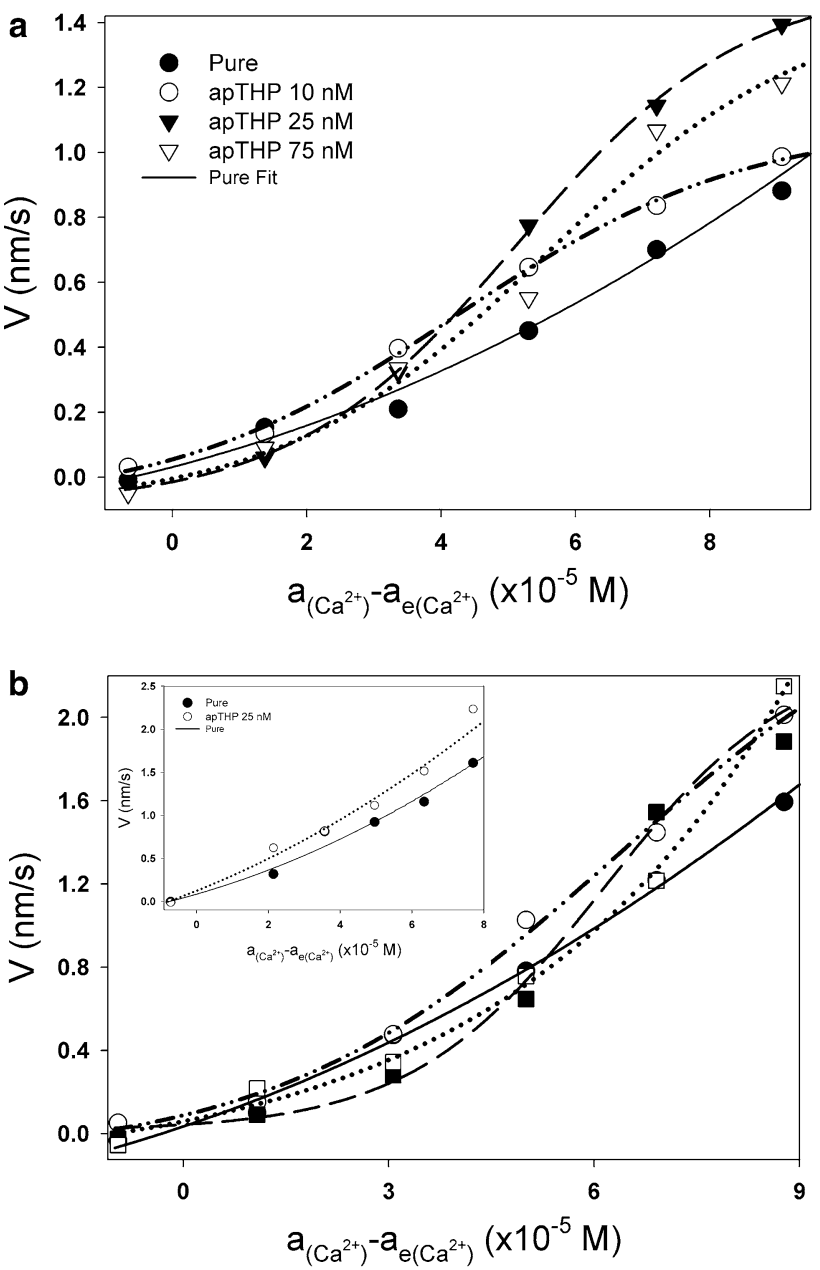

Fig. 5 The effect of apTHP on a the [021] step and $\mathbf{b}$ the [100] step of the (010) face of COM at an ionic strength of $0.05 \mathrm{M}$. For the [021] step, the addition of apTHP to the growth solution increases the step speed with respect to the pure system, while for the [100] step, the speed is depressed by apTHP at lower supersaturations and enhanced at higher supersaturations. The effect does not scale with apTHP concentration. Inset in $\mathbf{b}$ : The effect of apTHP on the [100] step of the (010) face of COM at an ionic strength of $0.15 \mathrm{M}$. Step speed is accelerated by apTHP

Figure 8 shows the observed temporal evolution of the (010) face following introduction of $5 \mathrm{nM}$ OPN. As with THP, the steps became less distinct after introduction of OPN (compare with Fig. 3). But unlike THP, step propagation was extensively inhibited at OPN levels of only $5 \mathrm{nM}$.

Synthetic Linear Aspartic-Acid(Asp)-Rich Peptides $\left[(\mathrm{DDDX})_{6} \mathrm{DDD}\right]$

Finally, we compare the results obtained with THP and OPN to those obtained with the 27-residue (DDDG) ${ }_{6}$ DDD peptide. As Fig. 9 shows, on the (010) face, these peptides created clusters similar to those observed with OPN on the 

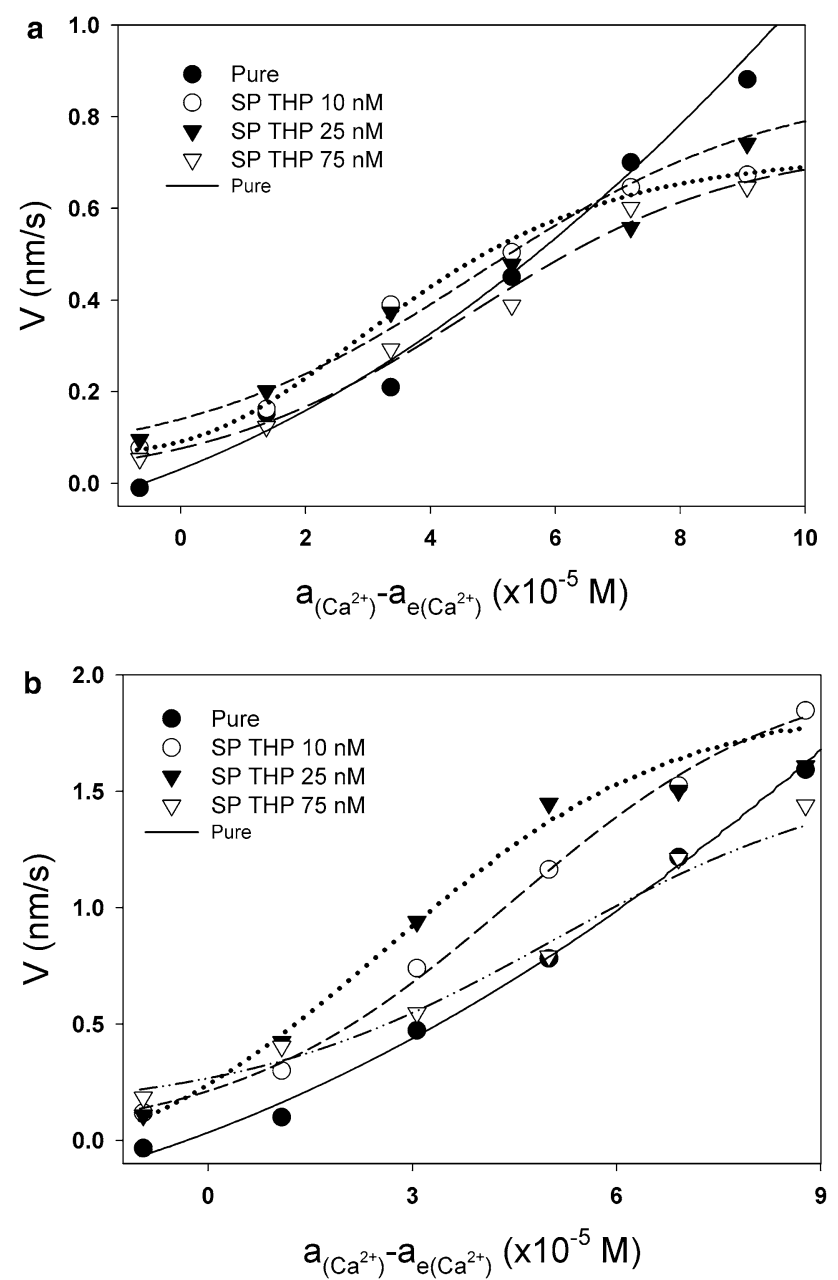

Fig. 6 The effect of spTHP on a the [021] step and b the [100] step of the (010) face of COM at an ionic strength of $0.05 \mathrm{M}$. For the [021] step, speed is accelerated at lower supersaturations and slight inhibition is observed at higher supersaturations, while for the [100] step, speed is accelerated, but as supersaturation increases the magnitude of the acceleration decreases. The effects do not scale with spTHP concentration

(101) face. As with OPN, the density of clusters increased with increasing peptide concentration, as did the average size. Once again, at low concentrations, the step morphology was largely unaffected. But as Fig. 10 shows, the step speed exhibits dual functionality on this face, as was the case for THP. Steps are inhibited at low supersaturation or high peptide levels and accelerated at higher supersaturation or low peptide levels (Fig. 10a, b, respectively).

In contrast to the discrete peptide aggregates seen on the (010) face, on the (101) face, the peptides made a continuous film, though at high concentrations some isolated aggregates also formed (Fig. 11). Even at low levels $(40 \mathrm{nM})$, this peptide caused significant inhibition of growth on this face at low supersaturation. There were wide regions of positive supersaturation where the step speed was near zero and steps exhibited extensive pinning (Fig. 11). But as Fig. 10a shows, with a small increase in supersaturation $(\Delta \sigma$ of $\sim 0.3$ ), inhibition was no longer observed and the steps recovered to their full speed.

While the results given here are for only one peptide concentration and for just the glycine compound, the data for all other concentrations and the results with (DDDS) $)_{6}$ DDD were found to be qualitatively similar, though the (DDDS) ${ }_{6}$ DDD concentrations needed to obtain the same density of clusters or levels of inhibition/acceleration were about 40 times lower. The complete data set and their analysis will be presented in a separate paper.

\section{Discussion}

The results presented here for OPN and the Asp-rich peptides are consistent with our previous work on these systems. We previously reported that OPN formed adsorbed clusters on the (101) face of COM [14]. We found that for a $1 \mathrm{nM}$ concentration of OPN, the clusters were about $1 \mathrm{~nm}$ in height, varied in diameter between about 10 and $50 \mathrm{~nm}$, and were spaced tens to hundreds of nanometers apart, with the density increasing over time. That study also showed that OPN strongly affected step morphology on the (010) face, with the steps showing extensive evidence for step pinning and with step growth inhibited to the point of complete cessation after $70 \mathrm{~min}$. However, at the concentrations used in that work, there was no apparent reduction in step speed on the (101) face, though quantitative measurements were not performed. Similarly, in a previous study looking at the effect of (DDDS) ${ }_{6} \mathrm{DDD}$ and (DDDG) ${ }_{6} \mathrm{DDD}$ [28], we found that the overall morphology of the (010) face following addition of this peptide was similar to that seen in Fig. 8 following OPN addition. However, the dependencies of morphology and step speed on peptide concentration were not determined and imaging was only performed at a low resolution. The results presented here fill these gaps by providing higher-resolution information about the protein-surface interactions and quantitative data on the changes in step kinetics.

Clearly, the modification in step kinetics by THP depends on the method by which it is extracted and there are also slight differences associated with changes in ionic strength. But the trend is clear. Despite the extensive coating by the proteins, the kinetics of step growth are generally accelerated by the presence of THP, although moderate inhibition can dominate on the (010) face at high THP concentrations and low supersaturations. These results provide clues to the possible roles of THP in calcium oxalate stone formation. The likely candidates are narrowed to the inhibition of crystal attachment and/or aggregation. The protein coating may account for the 
Fig. 7 Time sequence showing the development of OPN aggregates from initial (101) surface morphology (a) at OPN concentrations of $2 \mathrm{nM}(\mathbf{b}, \mathbf{c}, \mathbf{d})$ and $5 \mathrm{nM}(\mathbf{e}, \mathbf{f})$. Aggregates are elongated along the [010] direction. Image sizes and times are: as follows.

$0 \mathrm{nM}$ : a $9 \times 4.5 \mu \mathrm{m}, 0 \mathrm{~min}$. $2 \mathrm{nM}: \mathbf{b} 2 \times 1 \mu \mathrm{m}, 10 \mathrm{~min}$; c $3 \times 1.5 \mu \mathrm{m}, 30 \mathrm{~min}$; d $3 \times 1.5 \mu \mathrm{m}, 40 \mathrm{~min}$. $5 \mathrm{nM}$ : e $9 \times 4.5 \mu \mathrm{m}, 10 \mathrm{~min}$; f $5 \times 2.5 \mu \mathrm{m}, 20 \mathrm{~min}$
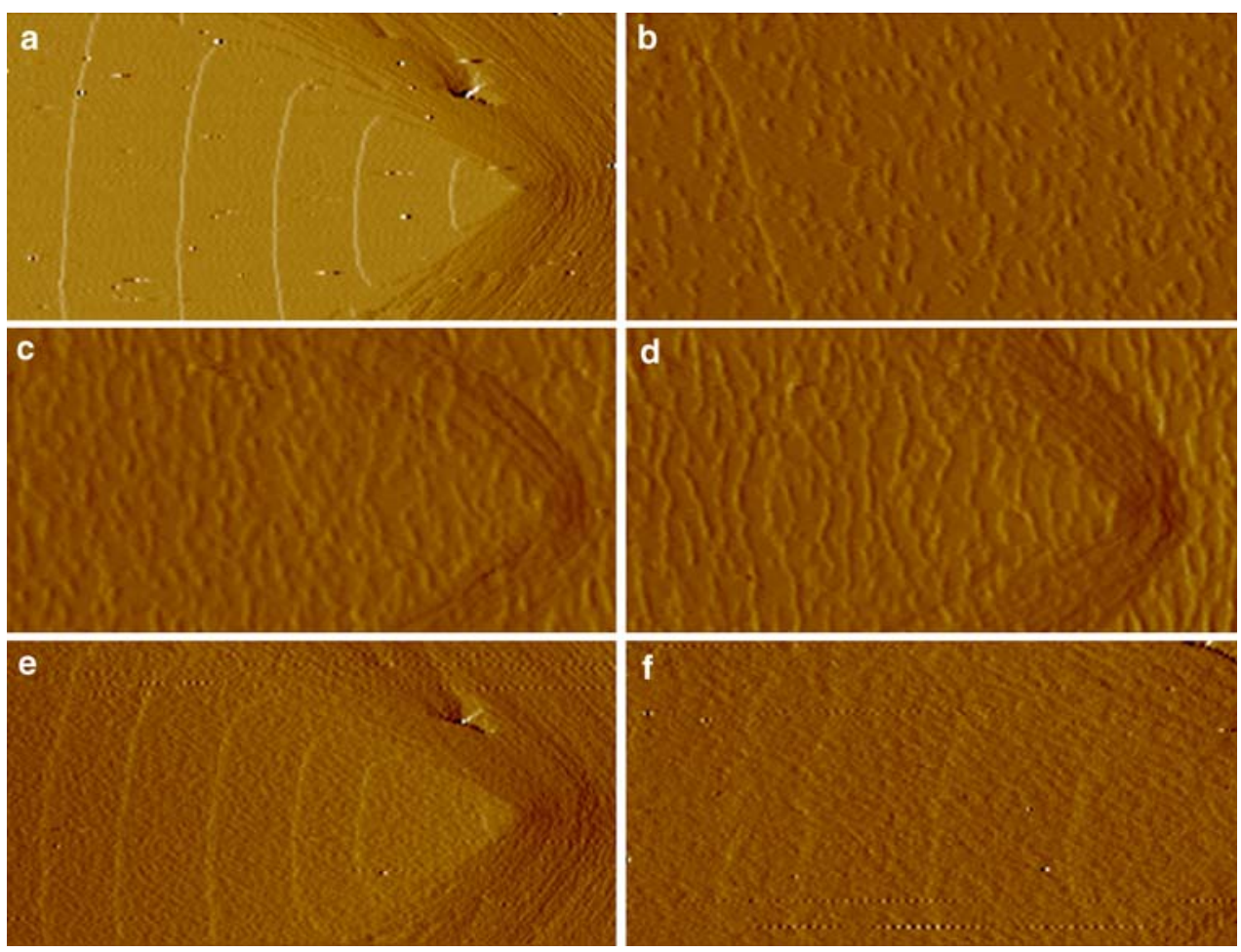

Fig. 8 Time sequence showing development of the (010) face at an OPN concentration of $5 \mathrm{nM}$. All image sizes are

$1.7 \times 1.7 \mu \mathrm{m}$ and times are $\mathbf{a}$ $0 \mathrm{~min}, \mathbf{b} 40 \mathrm{~min}$, c $50 \mathrm{~min}$, and d $70 \mathrm{~min}$
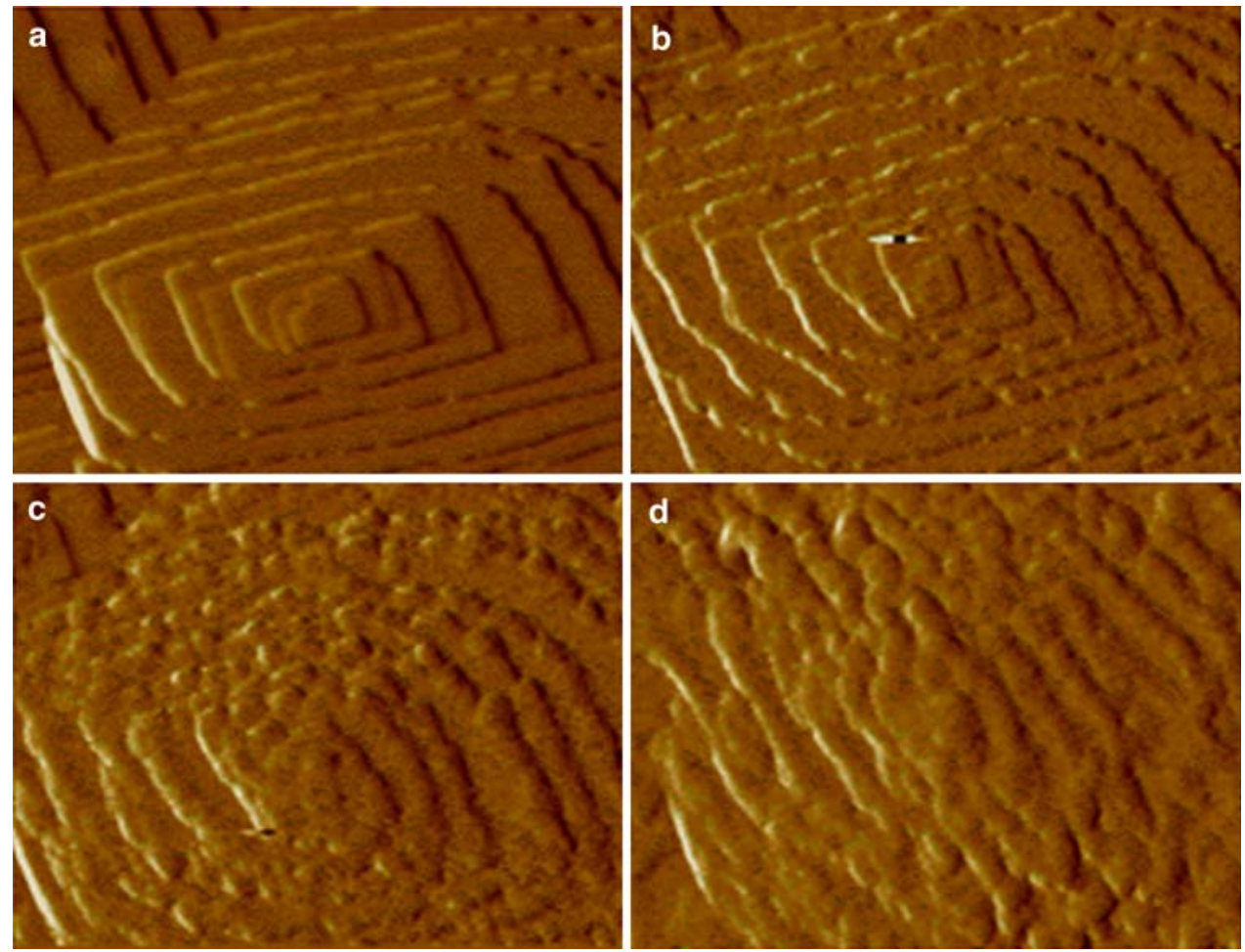

inhibitory effect on aggregation that has been observed previously $[9,10]$ as well as preventing attachment of crystals at cell surfaces [11-13]. Additionally, the presence of THP on the crystal surface may prevent the attachment of other nucleation-promoting macromolecules.
An obvious question raised by this study is whether the dual functionality that is observed to a limited extent for THP and to a much greater degree for the Asp-rich peptides has a useful function biologically. The ability of an organism to alternately accelerate or inhibit growth rates on 

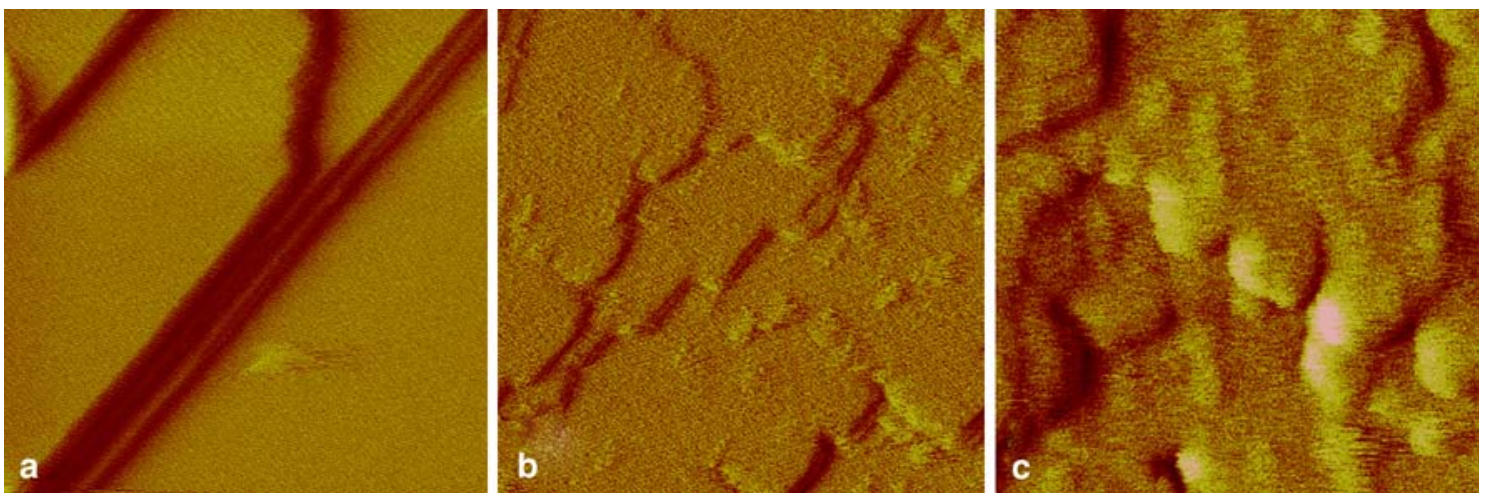

Fig. 9 Effect of (DDDG) ${ }_{6}$ DDD on growth morphology of the (010) face showing an increase in peptide cluster density with various peptide concentrations. Image sizes are all $150 \times 150 \mathrm{~nm}$
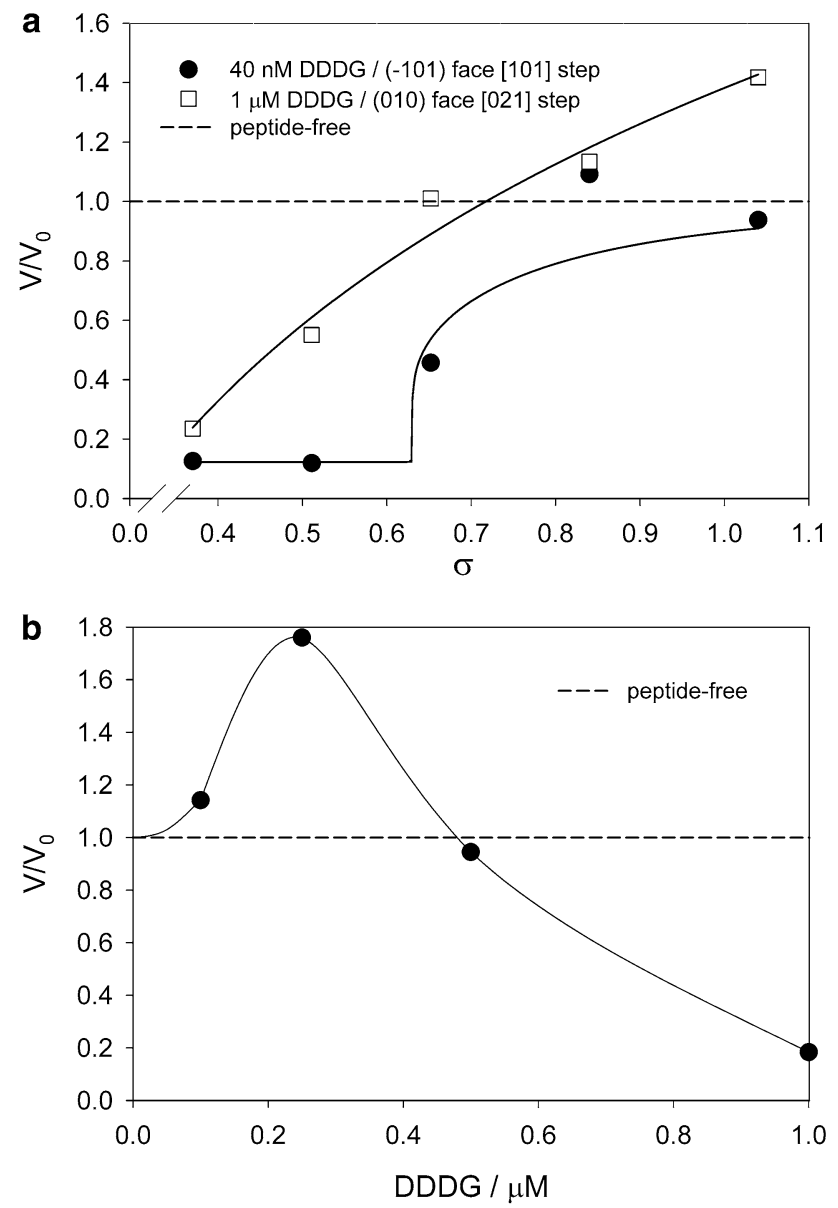

Fig. 10 a Dependence of the step speed normalized to the speed in the pure system on supersaturation in the presence of (DDDG) $)_{6} \mathrm{DDD}$ at a concentration of $100 \mathrm{nM}$ for the [101] step on the (101) face and $1 \mu \mathrm{M}$ for the [021] step on the (010) face. b Scaling of [100] step speed enhancement or inhibition with peptide concentration

specific faces with small changes in protein expression levels has obvious advantages during formation of functional tissues, for controlling both shape and overall size. But the advantage for limiting pathological mineralization is not at all clear and it may well be an unintended consequence of the inherent charged nature of COM crystal faces and the need for protein inhibitors to be rich in highly charged residues-particularly aspartic acid. The fact that the sequence of urinary OPN is close that of OPN from human bone provides some support for this suggestion.

Of the results presented here, the effect of THP on (101) growth kinetics are the most easily analyzed. As the data in Fig. 4 show, the step speed exhibits linear kinetics. This linear dependence is expected in the limit where growth is determined by the rate at which solute molecules leave the solution and attach to kink sites on the steps. In this case, one can write [29]:

$v=\omega \beta\left(a_{\left(\mathrm{Ca}^{2+}\right)}-a_{e_{\left(\mathrm{Ca} a^{2+}\right)}}\right)$

where $\omega$ is the specific molecular volume of COM, $\beta$ is the kinetic coefficient that governs solute attachment kinetics at the step edge, and $a_{\left(\mathrm{Ca}^{2+}\right)}-a_{e_{\left(\mathrm{Ca} a^{2+}\right.}}$ is the difference in actual and equilibrium calcium activities. Equation (3) is a simplification of an equation [30] that includes both calcium and oxalate activities; however, at our experimental conditions, where the activities of calcium and oxalate are nearly equal, Eq. (3) is a useful approximation. In this linear regime, the parameter $\beta$ is independent of calcium activity and exhibits an Arrhenius dependence on temperature: $\beta \propto e^{\left(-E_{a} / k_{B} T\right)}$. Consequently, the simplest interpretation of the data in Fig. 4 is that the activation barrier to attachment is reduced by the presence of the peptides. This same conclusion was reached from analysis of accelerated calcite growth in the presence Asp, polyAsp, Asp dipeptides, and (DDDX) ${ }_{6}$ DDD for $\mathrm{X}=\mathrm{S}$ and $\mathrm{G}$ [31]. Since activation energies for simple salts growing in solution all appear to be between about 25 and $35 \mathrm{~kJ} / \mathrm{mol}$ $[29,32]$, the level of acceleration seen here amounts to a change in activation energy of about $0.5 \mathrm{~kJ} / \mathrm{mol}$. Though it is a small fraction of the total barrier, its effect is amplified by the exponential dependence of $\beta$ on $\mathrm{E}_{\mathrm{a}}$. 
Fig. 11 Time sequence showing extensive step pinning during growth of the (101) face after introduction of (DDDG) ${ }_{6}$ DDD. Image sizes are all $150 \times 75 \mathrm{~nm}$. Frame interval is $8.4 \mathrm{~s}$

The inhibition of step growth on the (101) face by (DDDG) $)_{6}$ DDD and (DDDS) $)_{6}$ DDD is also easy to understand. The mechanism of inhibition is essentially the same as that which we reported for citrate inhibition [30, 33, 34] on this same face and is based on the classic model of Cabrera and Vermilyea [35]. The essential physics behind this model is the Gibbs-Thomson effect, which says that the equilibrium activity of a solute in solution is a function of the curvature of the solid-solution interface [36]. A highly curved surface is more soluble than a flat interface. The same is true for steps. A straight step can grow at a solute concentration at which a curved step cannot. Thus for any solute concentration, there is a critical radius of curvature such that less curved steps can grow but more curved steps cannot. That critical radius scales inversely with supersaturation.

In the case of the peptides, they adsorb to the surface and pin the advancing step. Between the pinning sites, the step segments bulge out as they try to advance and thus become curved. At low supersaturation, the critical radius of curvature is greater than the average spacing between the pinning sites and the step cannot advance. The result is that there is a region of positive supersaturation, known as the "dead zone," where no growth occurs. At high supersaturation, the critical radius drops until the steps can close around the pinning sites without exceeding the critical radius of curvature. Beyond that supersaturation, the step speed rapidly rises. The details of this analysis are beyond the scope of this paper, but we show elsewhere that the observed behavior can be quantitatively described with a slight modification of the original Cabrera-Vermilyea model to take into account the much slower adsorption rates expected for these much larger molecules. The solid curve through the data points for the (101) face in Fig. 10a shows the fit of the model to the data.

The effect of both the peptides and proteins on the kinetics of the (010) face is clearly more complicated. But the fact that both exhibit a transition from inhibition to acceleration with increasing supersaturation establishes a link between the two. To better understand the behavior, consider the dependence of step speed on peptide concentration shown in Fig. 10b. The step speed rises at a low peptide concentration and then drops towards zero as the peptide level continues to increase. Most of the step speed curves for this face in the presence of THP exhibit the same qualitative behavior: a rise to a maximum and then a decrease with increasing peptide concentration, but the magnitude of the effects is less
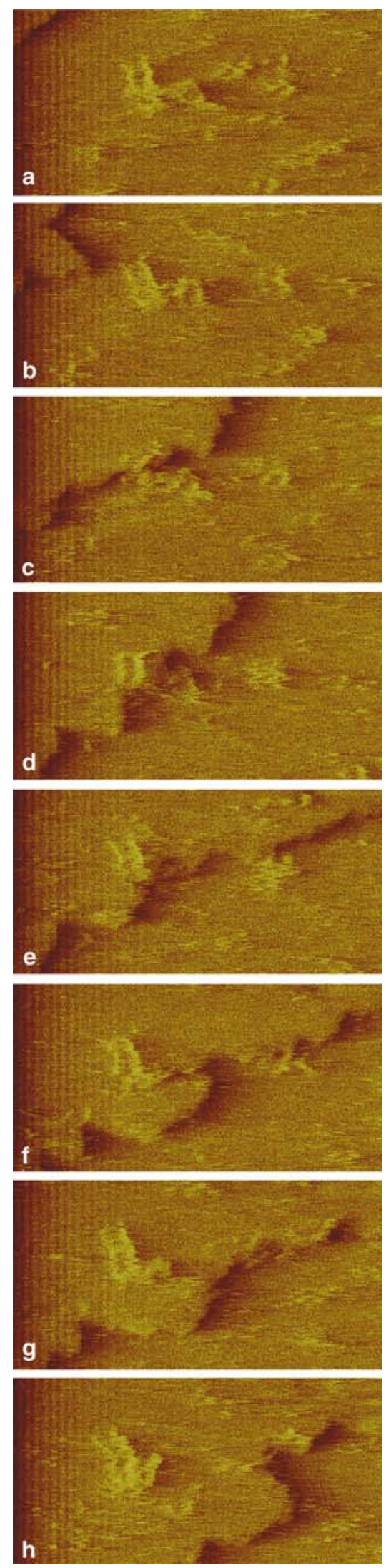
dramatic. More significantly, the behavior seen in Fig. 10b is precisely the behavior observed for calcite growth in the presence of D, poly-D, Asp dipeptides, and (DDDX) ${ }_{6} \mathrm{DDD}$ for $\mathrm{X}=\mathrm{S}$ and $\mathrm{G}[30]$. In the case of $\mathrm{D}_{\mathrm{n}}$, where $n$ was varied from 1 to 6 , analysis showed that the inhibition was well described by a simple Langmuir adsorption model in which peptides did not bind strongly enough to pin steps but just reduced the number of active kink sites [37]. As the images in Fig. 9 show, in the case of the peptides, the density of clusters increases with peptide level. And although the individual peptides apparently do not bind to the step, the clusters create a physical barrier to step propagation by blocking solute attachment. At a low peptide concentration, where the aggregate density is low, they provide little impedance to step motion. But at sufficiently high protein concentrations, the density of these clusters becomes too great for the steps to advance freely and they must squeeze between the many clusters. Thus growth is inhibited. Although we do not see clusters in the case of THP on the (010) face, by comparison to the calcite system and the COM-(DDDX) ${ }_{6} \mathrm{DDD}$ system, we can surmise that THP does not bind strongly to steps but presents a barrier to solute attachment at the steps.

To explain the acceleration, consider two observations. First, acceleration in the calcite system was shown to scale with both peptide charge and hydrophilicity and required a reduction in activation energy of as little as $0.015 \mathrm{~kJ} \times$ $\mathrm{mol}^{-1}$ for aspartic acid to as much as $2.5 \mathrm{~kJ}^{\circ} \times \mathrm{mol}^{-1}$ for an acidic $8-\mathrm{kDa}$ protein. From the dependence of calcite enhancement on peptide charge and hydrophilicity, Elhadj et al. [31, 37] argued that the peptides modify the local potential and solution structure near the crystal-liquid interface. Second, the COM (010) face is negatively charged due to the oxalate groups that protrude from the surface. But the peptides are highly anionic due to the 21 aspartic acid residues, each of which presents a carboxyl side group. One way the peptide clusters can form on this surface is to bring along an associated cloud of cations to balance the charge. This would also be true of individual solvated peptides in solution; to approach the surface, they would need to bring a counter-ion swarm along. This precise effect was recently predicted by a computational study of barite growth in the presence of aspartic acid [38]. The simulations predicted that the aspartic acid molecules would spontaneously associate with $\mathrm{Ba}^{2+}$ cations and assist their permeation through the solution as well as their attachment to the barite surface (cation bridging). Our conjecture is that this same process is taking place with COM. At low peptide concentrations where the density of clusters is too low to cause significant inhibition, the peptides in solution near the surface provide a supply of $\mathrm{Ca}^{2+}$ that enhances the growth rate. From the similarity of the results for the peptides and THP, by inference, we suggest that this picture should be extended to THP.
Even with the obvious source of both cations and anions to balance charge in these systems, the reason why proteins and peptides form surface aggregates when the electrostatic interactions are unfavorable is not obvious. One point to consider, however, is that, in solution, protein aggregation is driven by elimination of hydrophobic surfaces [39], not by specific bonds or electrostatic interactions. Moreover, as peptides get larger, they begin to exhibit properties of colloids, which also aggregate due to solvent effects, specifically osmotic forces associated with the increase in free volume of water brought about by condensation of the colloids [40]. When a surface is placed into a colloidal solution, even at colloid concentrations below the critical volume fraction, they will condense onto the surface for the same reason [41]. One should expect proteins to exhibit similar behavior. Consequently, the formation of OPN, THP, and (DDDX) 6 DDD aggregates on the charged surface of COM is likely driven by a combination of electrostatic and osmotic forces whose magnitudes and effects involve the complete set of constituents: water, solute ions, proteins, and mineral surface.

Acknowledgments This work was supported by grants from the National Institutes of Health (DK33501, DK61673 and DE03223). This work was performed under the auspices of the U.S. Department of Energy in part by Lawrence Livermore National Laboratory under Contract DE-AC52-07NA27344 and in part at the Molecular Foundry, Lawrence Berkeley National Laboratory, with support from the Office of Science, Office of Basic Energy Sciences, of the U.S. Department of Energy under Contract No. DE-AC02-05CH11231.

Open Access This article is distributed under the terms of the Creative Commons Attribution Noncommercial License which permits any noncommercial use, distribution, and reproduction in any medium, provided the original author(s) and source are credited.

\section{References}

1. Fleming DE, Van Riessen A, Chauvet MC, Grover PK, Hunter B, Van Bronswijk W, Ryall RL (2003) Intracrystalline proteins and urolithiasis: a synchrotron X-ray diffraction study of calcium oxalate monohydrate. J Bone Miner Res 18(7):1282-1291

2. Coe FL, Parks JH, Asplin JR (1992) The pathogenesis and treatment of kidney stones. N Engl J Med 327(16):1141-1152

3. Dussol B, Geider S, Lilova A, Leonetti F, Dupuy P, Daudon M, Berland Y, Dagorn JC, Verdier JM (1995) Analysis of the soluble organic matrix of five morphologically different kidney stones: evidence for a specific role of albumin in the constitution of the stone protein matrix. Urol Res 23(1):45-51

4. Kurutz JW, Carvalho M, Nakagawa Y (2003) Nephrocalcin isoforms coat crystal surfaces and differentially affect calcium oxalate monohydrate crystal morphology, growth, and aggregation. J Cryst Growth 255(3-4):392-402

5. Wesson JA, Ganne V, Beshensky AM, Kleinman JG (2005) Regulation by macromolecules of calcium oxalate crystal aggregation in stone formers. Urol Res 33(3):206-212 
6. Glauser A, Hochreiter W, Jaeger P, Hess B (2000) Determinants of urinary excretion of Tamm-Horsfall protein in non-selected kidney stone formers and healthy subjects. Nephrol Dial Transplant 15(10): 1580-1587

7. Shiraga H, Min W, VanDusen WJ et al (1992) Inhibition of calcium oxalate crystal growth in vitro by uropontin: another member of the aspartic acid-rich protein superfamily. Proc Natl Acad Sci USA 89(1):426-430

8. Worcester EM, Nakagawa Y, Wabner CL, Kumar S, Coe FL (1988) Crystal adsorption and growth slowing by nephrocalcin, albumin, and Tamm-Horsfall protein. Am J Physiol 255(6; Pt. 2):F1197-F1205

9. Hess B, Nakagawa Y, Coe FL (1989) Inhibition of calcium oxalate monohydrate crystal aggregation by urine proteins. Am J Physiol 257(1;Pt. 2):F99-F106

10. Hess B, Zipperle L, Jaeger P (1993) Citrate and calcium effects on Tamm-Horsfall glycoprotein as a modifier of calcium oxalate crystal aggregation. Am J Physiol 265(62):784-791

11. Kumar V, Farell G, Lieske John C (2003) Whole urinary proteins coat calcium oxalate monohydrate crystals to greatly decrease their adhesion to renal cells. J Urol 170(1):221-225

12. Kumar V, Pena de la Vega L, Farell G, Lieske John C (2005) Urinary macromolecular inhibition of crystal adhesion to renal epithelial cells is impaired in male stone formers. Kidney Int 68(4):1784-1792

13. Worcester EM (1994) Urinary calcium oxalate crystal growth inhibitors. J Am Soc Nephrol 5(5):S46-S53

14. Qiu SR, Wierzbicki A, Orme CA, Cody AM, Hoyer JR, Nancollas GH, Zepeda S, De Yoreo JJ (2004) Molecular modulation of calcium oxalate crystallization by osteopontin and citrate. Proc Natl Acad Sci USA 101(7):1811-1815

15. Ryall RL, Phulwinder KG, Thurgod LA, Chauvet MC, Fleming DE, van Bronswijk W (2007) The importance of a clean face: the effect of different washing procedures on the association of Tamm-Horsfall glycoprotein and other urinary proteins with calcium oxalate crystals. Urol Res 35(1):1-14

16. Sheng X, Jung T, Wesson JA, Ward MD (2005) Adhesion at calcium oxalate crystal surfaces and the effect of urinary constituents. Proc Natl Acad Sci USA 102(2):267-272

17. Hoyer JR, Asplin JR, Otvos L Jr (2001) Phosphorylated osteopontin peptides suppress crystallization by inhibiting the growth of calcium oxalate crystals. Kidney Int 60(1):77-82

18. Fields GB, Noble RL (1990) Solid-phase peptide synthesis utilizing 9-fluorenylmethoxycarbonyl amino acids. Int J Pept Protein Res 35(3):161-214

19. Hoyer JR, Otvos L Jr, Urge L (1995) Osteopontin in urinary stone formation. Ann N Y Acad Sci 760:257-265

20. Bleyer AJ, Hart TC, Shihabi Z, Robins V, Hoyer JR (2004) Mutations in the uromodulin gene decrease urinary excretion of Tamm-Horsfall protein. Kidney Int 66(3):974-977

21. Tamm I, Horsfall FL Jr (1950) Characterization and separation of an inhibitor of viral hemagglutination present in urine. Proc Soc Exp Biol Med 74:108-114

22. Fontan E, Briend E, Saklani-Jusforgues H, D'Alayer J, Vandekerckhove J, Fauve RM (1994) A 92-kDa human immunostimulatory protein. Proc Natl Acad Sci USA 91(18):8353-8357

23. Hession C, Decker JM, Sherblom AP, Kumar S, Yue CC, Mattaliano RJ, Tizard R, Kawashima E, Schmeissner U (1987)
Uromodulin (Tamm-Horsfall glycoprotein): a renal ligand for lymphokines. Science 237(4821):1479-1484

24. Cody AM, Horner HT, Cody RD (1982) SEM study of the fine surface features of synthetic calcium oxalate monohydrate crystals. Scan Electron Microsc 1982(1):185-197

25. Tomazic BB, Nancollas GH (1979) A study of the phase transformation of calcium oxalate trihydrate monohydrate. Investig Urol 16(5):329-335

26. Gilmer GH, Ghez R, Cabrera N (1971) Analysis of combined surface and volume diffusion processes in crystal growth. J Cryst Growth 8(1):79-93

27. Land TA, De Yoreo JJ, Lee JD (1997) An in situ AFM investigation of canavalin crystallization kinetics. Surf Sci 384(13):136-155

28. Wang L, Qiu SR, Zachowicz W, Guan X, De Yoreo JJ, Nancollas GH, Hoyer JR (2006) Constant composition studies verify the utility of the Cabrerea Vermilyea $(\mathrm{C}-\mathrm{-V})$ in explaining mechanisms of calcium oxalate monohydrate crystallization. Langmuir 22:7279-7285

29. De Yoreo JJ, Vekilov PG (2003) Principles of crystal nucleation and growth. Rev Mineral Geochem (Biomineralization) 54:57-93

30. Weaver ML, Qiu SR, Hoyer JR, Casey WH, Nancollas GH, De Yoreo JJ (2007) Inhibition of calcium oxalate monohydrate growth by citrate and the effect of the background electrolyte. J Cryst Growth 306:133-145

31. Elhadj S, De Yoreo JJ, Hoyer JR, Dove PM (2006) Role of molecular charge and hydrophilicity in regulating the kinetics of crystal growth. Proc Natl Acad Sci USA 103(51):19237-19242

32. Land TA, De Yoreo JJ (2000) The evolution of growth modes and activity of growth sources on canavalin investigated by in situ atomic force microscopy. J Cryst Growth 208(1-4):623-637

33. De Yoreo JJ, Qiu SR, Hoyer JR (2006) Molecular modulation of calcium oxalate crystallization. Am J Physiol 291(6, Pt. 2):F1123-F1131

34. Weaver M, Qiu SR, Hoyer JH, Casey WH, Nancollas GH, De Yoreo JJ (2006) Improved model for inhibition of pathological mineralization based on citrate-calcium oxalate monohydrate interaction. ChemPhysChem 7:2081-2084

35. Cabrera N, Vermilyea DA (1958) Growth of crystals from solution. In: Growth and perfection of crystals, proceedings of the international conference, Cooperstown, NY, pp 393-410

36. Chernov AA (1984) Modern crystallography III: crystal growth. In: Cardona M, Fulde P, Queisser HJ (eds) Springer series in solid state sciences. Springer, Berlin

37. Elhadj S, Salter EA, Wierzbicki A, De Yoreo JJ, Han N, Dove PM (2006) Peptide controls on calcite mineralization: polyaspartate chain length affects growth kinetics and acts as a stereochemical switch on morphology. Cryst Growth Des 6(1):197-201

38. Piana S, Jones F, Gale JD (2007) Aspartic acid as a crystal growth catalyst. CrystEngComm 9(12):1187-1191

39. Chothia C, Janin J (1975) Principles of protein-protein recognition. Nature 256(5520):705-708

40. Cheng Z, Russel WB, Chaikin PM (1999) Controlled growth of hard-sphere colloidal crystals. Nature 401(6756):893-895

41. Van Blaaderen A (2004) Colloids under external control. MRS Bull 29(2):85-90 